\title{
Analytical calculation of intrinsic shielding effectiveness for isotropic and anisotropic materials based on measured electrical parameters
}

\author{
M. Kühn ${ }^{1}$, W. John ${ }^{2}$, and R. Weigel ${ }^{3}$ \\ ${ }^{1}$ AUDI AG, 85045 Ingolstadt, Germany \\ ${ }^{2}$ SIL System Integration Laboratory GmbH, Technologiepark 32, 33100 Paderborn, Germany \\ ${ }^{3}$ Lehrstuhl für Technische Elektronik, Friedrich-Alexander-Universität Erlangen-Nürnberg, Cauerstrasse 9, 91058 Erlangen, \\ Germany
}

Correspondence to: M. Kühn (michael.kuehn@audi.de)

Received: 29 January 2014 - Accepted: 14 September 2014 - Published: 10 November 2014

\begin{abstract}
This contribution contains the mechanisms for calculation of magnetic shielding effectiveness from material samples, based on measured electrical parameters. For this, measurement systems for the electrical conductivity of high and low conductive material samples with respect to the direction of current flow are presented and discussed. Also a definition of isotropic and anisotropic materials with electrical circuit diagrams is given. For prediction of shielding effectiveness for isotropic and anisotropic materials, several analytical models are presented. Also adaptions to gain a near field solution are part of this contribution. All analytical models will also be validated with an adequate measurement system.
\end{abstract}

\section{Introduction}

In common, shielding effectiveness of material samples is measured with a capacitive coupled Transmission-LineHolder (TLH) based on ASTM D4935-10 standard. This TLH is a widened coaxial cable, where the surface of the material sample is coupled perpendicular to the inner and outer conductor. In other words, the coaxial cable is separated by the material sample. This means, that the field vectors are oriented parallel to the surface of the material sample and the far field shielding effectiveness is measured. In theory this is described by the Impedance Concept of Schelkunoff (see Schelkunoff, 1938) with the physical processes of reflection, transmission and absorption of electromagnetic waves at material boundaries. In a vehicle with combustion engine or electrical drive train are in most of the cases electromagnetic source and victim placed in a closed distance $(r)$ to each other, that the near field condition $r \ll \frac{\lambda}{2 \pi}$ is fulfilled (Schwab and Kürner, 2011). Separation of the field components (electrical and magnetic field) for analysis of shielding effectiveness is necessary. The focus in this contribution is laid on magnetic shielding effectiveness in the near field area, reasoned by high switching currents generated by the power electronics in an electrified vehicle. Strong magnetic fields with fundamental frequencies in the lower frequency range $(10 \mathrm{kHz}$ to $20 \mathrm{kHz})$ with a wide spectrum up to the $\mathrm{MHz}$ range are the result, see Michel (2011). Based on measured electrical parameters (electrical conductivity), a method will be presented to calculate magnetic shielding effectiveness of material samples in the near field area. For isotropic materials (i.e. Aluminum) there are several analytical models in the literature available. Among others a modification of Schelkunoff's Impedance Concept (see Wolfsperger, 2008) for the near field area and the analytical model from Moser (see Moser, 1967). Also considered in this contribution, materials like Carbon Fibre Reinforced Polymers (CFRP). Anisotropy of CFRP will be discussed with electrical circuit diagrams. An approach for calculating magnetic shielding effectiveness based on measured electrical properties for the near field area will also be presented. 

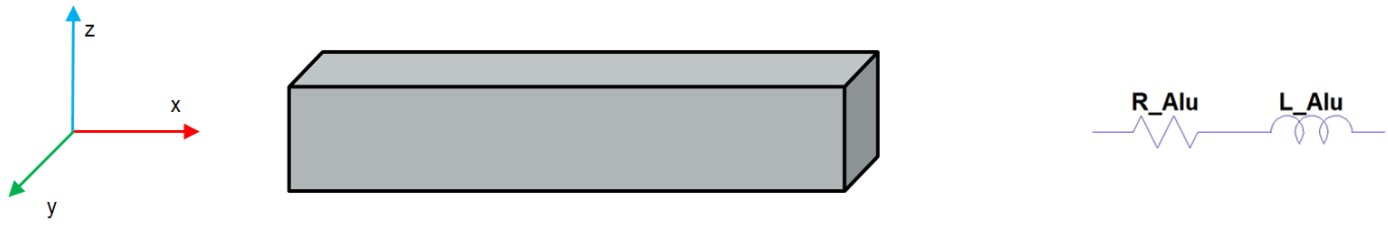

Figure 1. Isotropic material and equivalent circuit diagram.
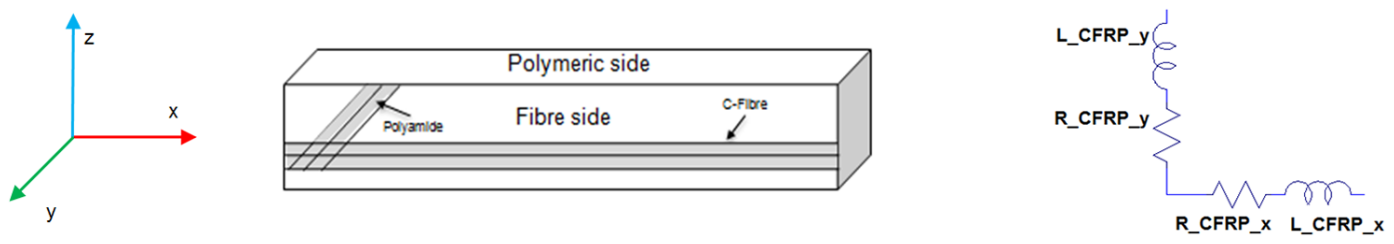

Figure 2. Anisotropic material and equivalent circuit diagram for $x$ and $y$ direction.

\section{Near field shielding effectiveness}

This section describes the physical processes accountable for shielding of electrical and magnetic fields in the frequency domain. First of all, a definition of near field is necessary. Fields are defined in the frequency range as near fields, when the wavelength $\lambda$ is large compared to the distance $(r)$ from field source to observing point. The following condition (1) must be fulfilled (Schwab and Kürner, 2011):

$r \ll \frac{\lambda}{2 \pi}$

The near field shielding effectiveness of electrical fields on enclosure level is nearly infinity and the shielding process is described by the Faraday cage. The electrical field bears a force on charge carriers in a conductive material, Near field shielding effectiveness should be considered separately for the electrical and magnetic field. At first, the physical processes for electrical shielding effectiveness will be described.

\subsection{Electrical fields}

A finite piece of material (thickness $d$ and area $A=a \cdot b$ ) is penetrated by an incident electrical field, displacement currents transported by the electrical field lines are closing in the conductive shield material. A part of electrical field lines encompasses the shield and closes behind it. So, electrical shielding effectiveness on material level depends strongly on the geometry of the shield (area $A$ ) and the distance from the source to the shield $(r)$. For an enclosure or housing, shielding process is described by the Faraday cage structure (principle: charge carrier separation). In theory electrical shielding effectiveness of infinite extensive material samples is nearly infinity.

\subsection{Magnetic fields}

The magnetic field will be shielded respectively reduced by two physical processes (see Fig. 1), the generation of eddy currents in the shield and skin effect. The incident magnetic field leads to a voltage in the shield, which results in eddy currents in the shield. These eddy currents are generating an inverse oriented magnetic field, which weakens the incident magnetic field. The skin effect describes the reduction of the current density of a conductor or shield by raising frequency. In summary, the magnetic shielding effectiveness depends on the shield material $\left(\sigma, \mu_{r}\right)$ and the thickness of the shield.

\section{Material catalog and measurement methods}

The material catalog contains two types of materials:

- Isotropic materials

- Anisotropic materials

which will be described in the following subsections. Also two different methods for measuring conductivity will be presented and discussed.

\subsection{Isotropic materials}

An isotropic material is defined as a material with a constant electrical conductivity $(\sigma)$ in all three spatial directions $(x, y, z)$. Figure 1 shows an isotropic material (i.e. Aluminum) and the corresponding equivalent circuit diagram, which is valid for all three spatial directions.

The equivalent circuit diagram consists of a resistor and an inductance in series, which are geometry dependent.

\subsection{Anisotropic materials}

An anisotropic material distinguishes from an isotropic material in that way, that the electrical conductivity is not constant 

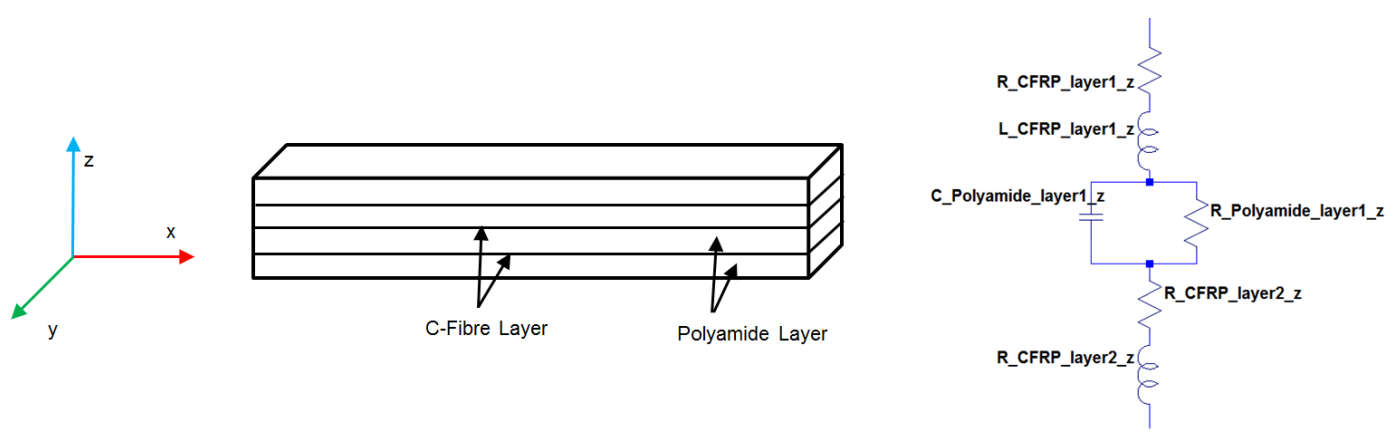

Figure 3. Anisotropic material and equivalent circuit diagram for $z$ direction.

in all three spatial directions. For example CFRP (Carbon Fibre Reinforced Polymers) which consists of C-fiber layers $\left(90^{\circ}\right.$ woven) and a Polyamide 6 matrix. Obviously, the conductivity should be similar in fiber direction and differs through the layered structure. Both is visualized with equivalent circuit diagrams, in Fig. 2 for $x, y$ direction and Fig. 3 for $z$ direction.

In fiber direction, the equivalent circuit diagram corresponds to the isotropic material and consists of a resistor and inductance in series.

Through the layered structure (assumption: alternating layers of C-Fiber and Polyamide 6) the equivalent circuit diagram also contains a capacitor for the non conductive Polyamide 6 and parallel a high ohmic resistor. The C-Fiber is also modeled by a resistor and inductance in series.

\subsection{Four-Point-Probe-Measurement}

Four-Point-Probe-Measurement method could be used for measurement of electrical conductivity at $0 \mathrm{~Hz}$ (DC). A known current $\left(I_{\text {in }},(1),(4)\right)$ was fed into the material sample (MUT) and the voltage drop ( $\left.U_{\text {meas }},(2),(3)\right)$ was measured. The measurement setup is shown in Fig. 4.

With the input current $I_{\text {in }}$, the voltage drop $U_{\text {meas }}$ and the geometry of the material sample (area $A$, length $l$ ) it is possible to calculate the electrical conductivity $\sigma$ with the following Eqs. (2) based on the specific resistance $\rho$ (Bowler, 2006):

$\rho=\frac{U_{\text {meas }}}{I_{\text {in }}} \cdot \frac{A}{l}$

$\sigma=\frac{1}{\rho}$

Four-Point-Probe-Measurement method was performed for Aluminium in $x, y$ and $z$ direction CFRP in $x$ and $y$ direction. For CFRP was silver paste used for better connection of the electrodes and a homogenous current distribution in the MUT. Electrical conductivity of Aluminum is constant for all three spatial directions: $\sigma_{\mathrm{Alu}, x, y, z} \approx 37.9 \mathrm{MS} / \mathrm{m}$ (comparison to literature value: $37.7 \mathrm{MS} / \mathrm{m}$, see Vinaricky et al., 2002). Electrical conductivity in $x$ and $y$ direction of

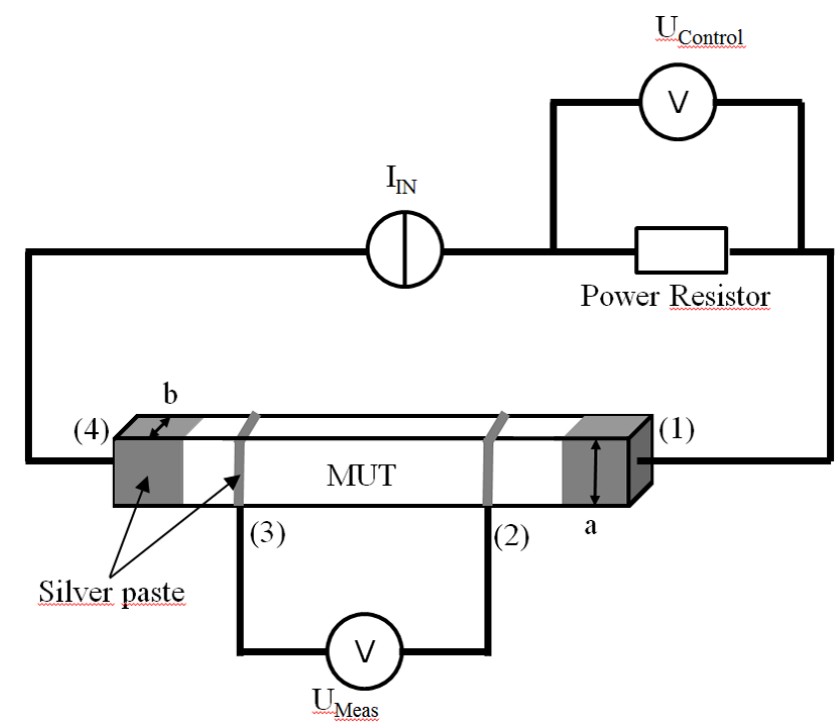

Figure 4. 4-Point-Probe-Measurement method.

$\sigma_{\mathrm{CFRP}, x, y} \approx 12.54 \mathrm{kS} / \mathrm{m}$ was measured for CFRP. Additional is to say, that the conductivity of CFRP depends strongly of the quality and consistency of the MUT like fibre position and fibre concentration.

\subsection{Capacitive measurement method}

The missing $z$ direction of an anisotropic material respectively of CFRP could be measured by a capacitive measurement method with an Impedance Analyser. This measurement method is usually used for measuring permittivity of dielectric materials respectively for materials with a very low conductivity (Impedance Spectroscopy). Also preparation of MUT with silver paste was necessary, for a better connection of the electrodes. With a Four-Point-Probe measurement were the capacitance $\left(C^{\prime}\right)$ and the real part of the conductance $\left(G^{\prime}\right)$ measured. Figure 5 shows the connection of the electrodes and the preparation of the MUT.

Based on geometry of the MUT, the measured capacitance $C^{\prime}$ and conductance $\left(G^{\prime}\right)$ calculation of electrical 


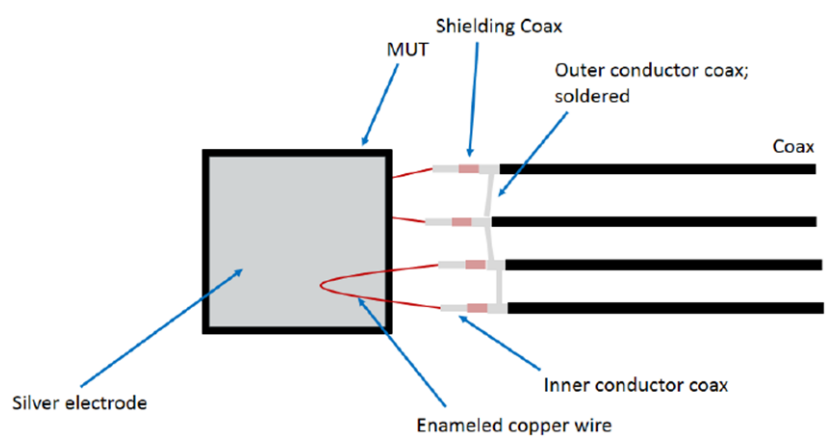

Figure 5. Capacitive measurement method and preparation of the MUT.

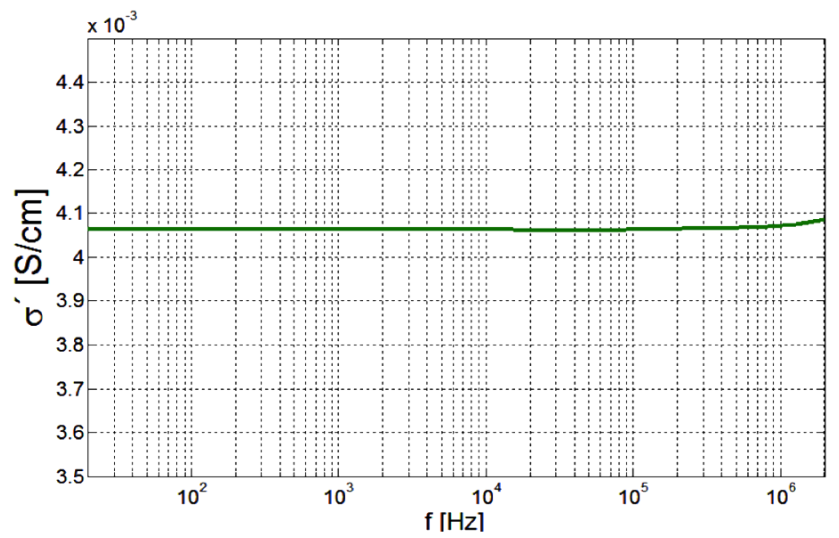

Figure 6. Measurement results of CFRP in $z$ direction with capacitive method.

conductivity is possible, see Eqs. 3. This method is only suitable for materials with a very low conductivity, because higher conductive materials occur a short between the electrodes respectively the capacitor. The measurement was performed for CFRP in $z$ direction in the frequency range $40 \mathrm{~Hz}<f<10 \mathrm{MHz}$.

$$
\begin{aligned}
C_{0} & =\frac{\epsilon_{0} \cdot A}{d} \\
\epsilon^{\prime} & =\frac{C^{\prime}}{C_{0}} \\
C^{\prime \prime} & =\frac{G^{\prime}}{\omega} \\
\epsilon^{\prime \prime} & =\frac{C^{\prime \prime}}{C^{\prime \prime} \cdot \epsilon^{\prime}} \\
\sigma^{\prime} & =\epsilon \cdot \epsilon^{\prime \prime} \cdot \omega
\end{aligned}
$$

The measurement results in the considered frequency range is shown in Fig. 6.

The electrical conductivity measured with the capacitive method was for CFRP in $z$ direction $\sigma_{\mathrm{CFRP}, z} \approx 0.41 \mathrm{~S} / \mathrm{m}$. The results have shown, that single Carbon fibre layers are electrically insulated. An assumption, that CFRP is a multilayered material with insulated layers could be made. All presented

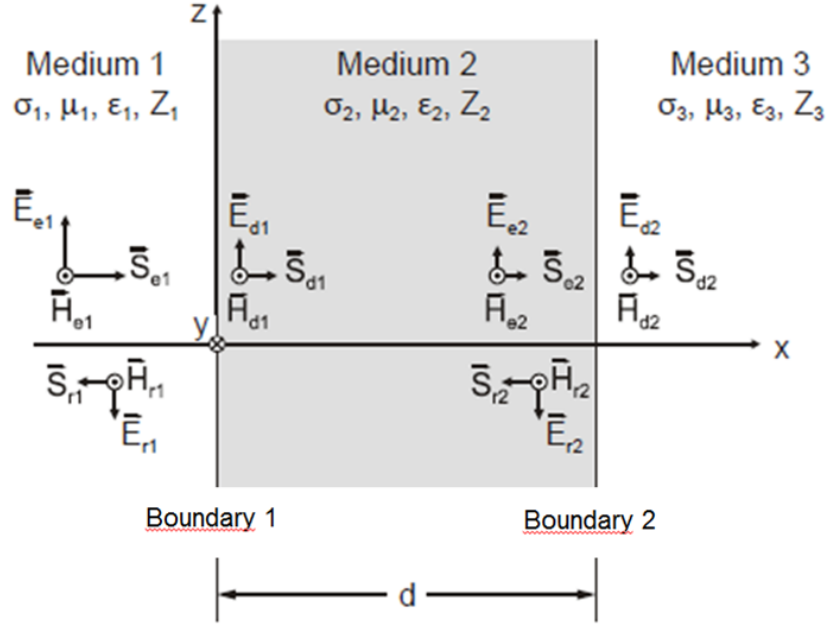

Figure 7. Impedance Concept by Schelkunoff, Wolfsperger (2008).

measurement results in this chapter will be used in the next chapter for the analytical calculation of the shielding effectiveness.

\section{Analytical models for calculating shielding effective- ness}

This section describes analytical models for calculating shielding effectiveness of isotropic and anisotropic materials.

\subsection{Impedance Concept by Schelkunoff}

The Impedance Concept from Schelkunoff (see Schelkunoff, 1938) describes the physical processes reflection, absorption and transmission of plane waves at material boundaries. This model is based on transmission line theory and the change of wave impedance at material boundaries. It is valid for isotropic, single layered materials. Figure 7 visualises the impedance concept for a plane wave (TEM-Wave). With the following equations calculation of free space impedance $Z_{0}$ (Eq. 4) and impedance of the material sample $Z_{M}$ (Eq. 5) is possible. $Z_{M}$ depends on the permeability $\mu$, the permittivity $\epsilon$, the frequency $\omega$ and the electrical conductivity $\sigma$. For dia- and paramagnetic materials, like Aluminum or CFRP, is $\mu_{r} \approx 1$. With this configuration, calculation of far field shielding effectiveness for material samples is possible.

$Z_{0}=\sqrt{\frac{\mu_{0}}{\epsilon_{0}}}$

$Z_{M}=\sqrt{\frac{i \omega \mu}{\sigma+i \omega \epsilon}}$

As mentioned above, shielding effectiveness in the near 


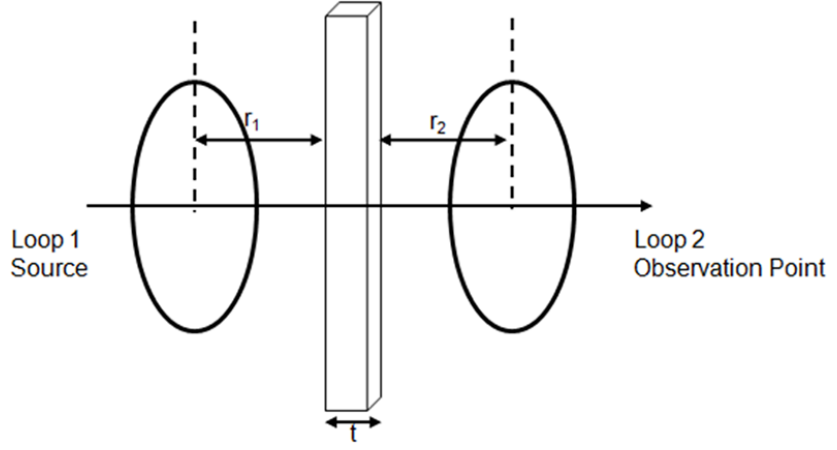

Figure 8. Analytical Model from Moser, Moser (1967).

field area is more interesting respectively the use case in a vehicle. In Wolfsperger (2008) is an approach for calculation shielding effectiveness with the Impedance Concept by Schelkunoff (Schelkunoff, 1938) in the near field area. This approach is based on substitution of the free space impedance $Z_{0}$ by the impedance of an loop antenna/dipole antenna in the near field area ( $Z_{L}$ Eq. 14; $Z_{C}$ Eq. 7), Wolfsperger (2008).

$Z_{L}=\frac{Z_{0} \cdot \lambda}{2 \pi \cdot r}$

$Z_{C}=\frac{Z_{0} \cdot 2 \pi \cdot r}{\lambda}$

\subsection{Model from Moser}

The model from Moser (see Moser, 1967) is valid for the calculation of magnetic shielding effectiveness in the near field area. The schematic draw in Fig. 8 shows two coils (source and observation point) seperated by an infinite material sheet.

The model contains an exact solution of the vector wave Eq. (8).

$\nabla^{2} \vec{A}+k_{0}^{2} \cdot \vec{A}=0$

Validation with a measurement and comparison to the Impedance Concept by Schelkunoff was done by Moser (1967). For validation of the measurement method, analog to the setup in Fig. 8, comparison will be done again in Sect. 5.

\subsection{Enhanced impedance concept for multi layered structures}

In Imo et al. (1993) is a model for calculation shielding effectiveness of a parallel-plate-shield (see Fig. 9). This parallelplate-shield consists of two infinite extensive plates with a defined thickness $(d)$ and a distance to each other of $2 \cdot x_{0}$. This shield structure is penetrated by a plane wave (TEMWave) from outside and shielding effectiveness is calculated in the middle of the plates with a chain matrix method. Every single matrix could be understand as a quadripole, which

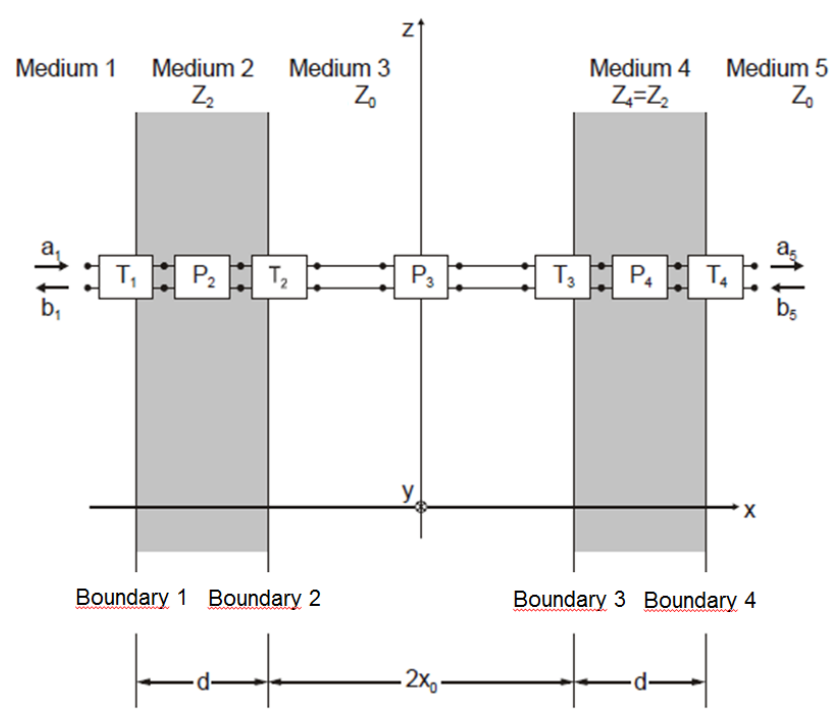

Figure 9. Enhanced Impedance Concept, Wolfsperger (2008).

consists of transmission- and reflection factors for every material and material boundary. The chain matrices could be enhanced for multilayered shields easily, also for CFRP materials. In the first approximation every Carbon fibre layer with $90^{\circ}$ fibre direction could be seen as isotropic (see conductivity measurements in Sect. 3.3) and electrically insulated from the next Carbon fibre layer by a Polyamide layer. The validity of this approximation needs to ensured with measurements, see Sect. 5. The measurement results in Sect. 3.4 have shown a very low conductivity in $z$ direction through the layered structure for CFRP $\left(\sigma_{\mathrm{CFRP}, z} \approx 0.41 \mathrm{~S} / \mathrm{m}\right)$.

For calculation of shielding effectiveness in the near field area of multilayered materials (e.g. CFRP) adaptions of the model from Imo et al. (1993) were necessary:

- Shielding effectiveness behind the shield respectively multilayered material

- Solution for near field sources

Due to the simple structure of the quadripoles and the matrices, calculation of shielding effectiveness behind the screen was easy. In the following, the method will be called: Chain matrix method for multilayered materials (CMM). For the following example $(n=2$ layers of Carbon fibre and $m=1$ layer of Polyamide, see Fig. 10) the chain matrix looks like (Eqs. 9 and 10):

$\left[\begin{array}{l}a_{1} \\ b_{1}\end{array}\right]=\left[T_{1}\right]\left[P_{2}\right]\left[T_{2}\right]\left[P_{3}\right]\left[T_{3}\right]\left[P_{4}\right]\left[T_{4}\right]\left[\begin{array}{c}a_{5} \\ 0\end{array}\right]$

$\left[\begin{array}{l}a_{1} \\ b_{1}\end{array}\right]=\left[\begin{array}{ll}s & t \\ u & v\end{array}\right]\left[\begin{array}{c}a_{5} \\ 0\end{array}\right]$

The parameter $b_{5}=0$ because there are no reflections behind the material, there is just free space. Shielding effectiveness 


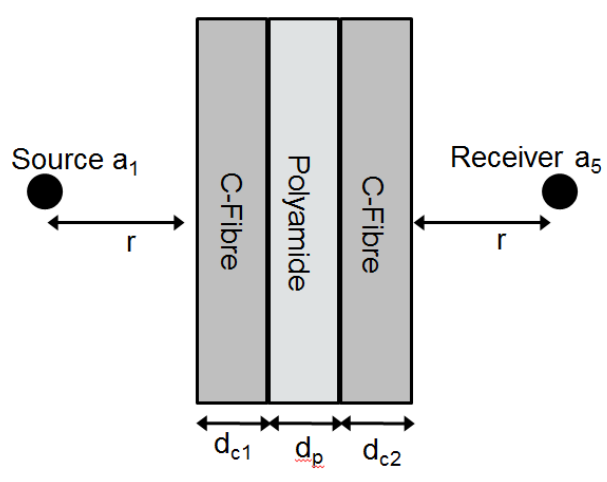

Figure 10. Example for the chain matrix method for calculation shielding effectiveness of CFRP.

could be calculated by the following Eq. (11):

$s^{-1}=\frac{a_{5}}{a_{1}}$

For calculation of the near field shielding effectiveness, a detailed view on the transmission matrix $\left(T_{1}\right.$, see Eq. 12) respectively there elements $\left(d_{I I, 2}\right.$ transmission factor equation and $r_{I I, 2}$ reflection factor) was necessary. As described in Sect. 4.1 a substitution of the free space impedance $\left(Z_{0}\right)$ with the impedance of the inductive field $\left(Z_{L}\right)$ or capacitive field $\left(Z_{C}\right)$ it is also possible to get the near field solution.

$\left[T_{1}\right]=\frac{1}{d_{I I, 1}}\left[\begin{array}{cc}1 & r_{I I, 1} \\ r_{I I, 1} & 1\end{array}\right]$

$d_{I I, 1}=\frac{2 \cdot Z_{L, C}}{Z_{M}+Z_{L, C}} \quad r_{I I, 1}=-\frac{Z_{M}-Z_{L, C}}{Z_{M}+Z_{L, C}}$

$Z_{L}=\frac{Z_{0} \cdot \lambda}{2 \pi \cdot r} \quad Z_{C}=\frac{Z_{0} \cdot 2 \pi \cdot r}{\lambda} \quad Z_{M}=\sqrt{\frac{i \omega \mu}{\sigma+i \omega \epsilon}}$

The following Fig. 11 shows the results for the magnetic near field shielding effectiveness for $m=1,5,10,20$ layers of Polyamide respectively $n=m+1$ layers of Carbon fibre.

For raising $m$ or $n$ magnetic shielding effectiveness in the near field is also raising. In the lower frequency range $(<10 \mathrm{kHz})$ is no shielding functionality available, this is reasoned by the low conductivity of the Carbon fibre layer $\left(\sigma_{\mathrm{CFRP}, x, y} \approx 12.5 \mathrm{kS} / \mathrm{m}\right)$.

\section{Validation with measurements}

For validation of the previous presented models a suitable measurement method was designed, The previous presented models need to be validated with measurements. For this, a suitable measurement system was designed. The measurement system with two coils (source and observation point)

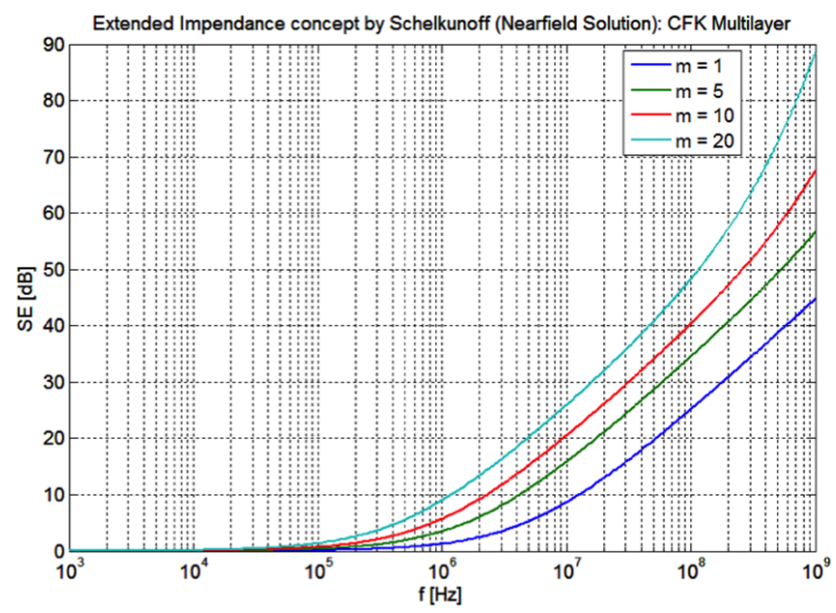

Figure 11. Shielding effectiveness calculated with chain matrix method for $n=m+1$ layers Carbon fibre.

wounded on a PVC tube (radius $d=0.0375 \mathrm{~m}, N=5$ Windings, distance between the coils $D=0.085 \mathrm{~m}$ ) was inspired by Fig. 8 . The material sample separates the coils in the middle. Shielding effectiveness $(S E)$ could be calculated after measuring the induced voltage in the observation coil with two different configurations ( $U_{\text {indmax }}$ without material sample, $U_{\text {indsample }}$ without material sample), see Eq. (15):

$S E=20 \cdot \log \frac{U_{\text {indmax }}}{U_{\text {indsample }}}$

The MUT (Aluminum, thickness $d=300 \mu \mathrm{m}$ ) was chosen with adequate area $(A=0.3 \mathrm{~m} \cdot 0.2 \mathrm{~m})$ to minimize the scattered field (magnetic field outside the MUT), which would distort the measurement result. The frequency range were the measurement was performed: $500 \mathrm{~Hz}<f<300 \mathrm{kHz}$. The comparison with the Impedance Concept of Schelkunoff (near field modification), the model from Moser and the measurement for Aluminum is shown in Fig. 12.

The measurement (blue crosses) and the analytical model from Moser (green curve) show, especially in the lower frequency range $(f<1 \mathrm{kHz})$, a very good match. The Impedance Concept from Schelkunoff (red curve) matches by $f>2.5 \mathrm{kHz}$ the measurement or the model from Moser. The frequency range $f<2.5 \mathrm{kHz}$ is not described correctly by the Impedance Concept, negative values are not plausible. For $f>225 \mathrm{kHz}$ a deviation from measurement to the models is recognisable, this is reasoned by the limits of the measurement system (dynamic problem). The same measurement was also performed with a CFRP Material. The measurement was compared with the results of the Chain Matrix Method. Parametrisation of the model was appropriate to the corresponding CFRP material. Figure 13 shows the measurement results compared to the Chain Matrix Method results. 


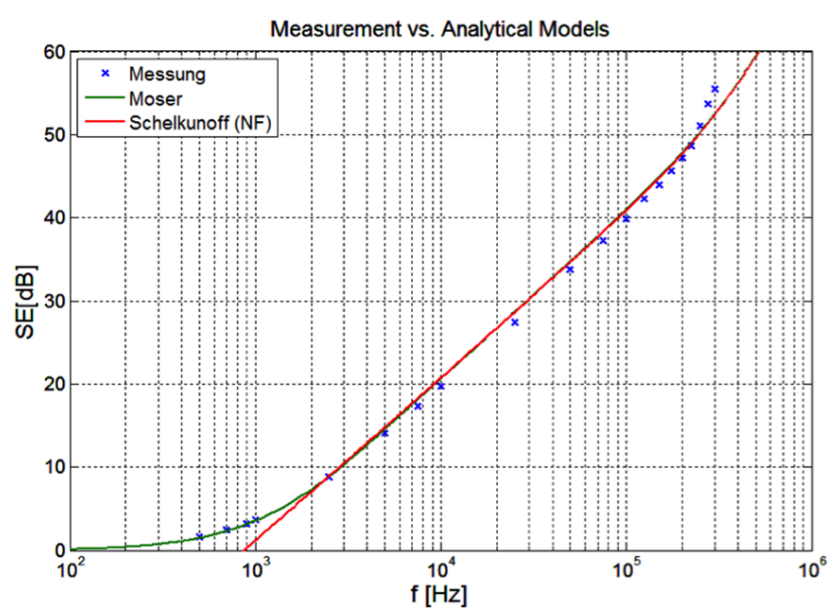

Figure 12. Comparison of Schelkunoff's Impedance Concept and the model from Moser with a measurement.

\section{Conclusions}

In this contribution, the electrical behaviour of isotropic (Aluminum) and anisotropic (CFRP) based on electrical circuit diagrams, was analysed. Also measurement techniques for low and high conductive materials, with respect to the current flow direction, were presented. Those measurements have shown, that CFRP could be handled as multilayered material (Carbon fibre layers insulated by Polyamide). For prediction of magnetic shielding effectiveness were different analytical models presented and discussed, the Impedance Concept by Schelkunoff and the analytical model from Moser. The Impedance Concept was adapted for the near field solution and the model from Moser contains an exact solution for the magnetic near field area. To calculate shielding effectiveness of anisotropic materials, the enhanced Impedance Concept for calculation of shielding effectiveness of parallelplate-shields, was modified and adopted. Validation of all analytical models was done with an adequate measurement setup, which was inspired by the model from Moser. Measurement results showed a good match with the analytical model from Moser in the considered frequency range. The Impedance Concept (near field solution) correlated for $f>2.5 \mathrm{kHz}$ with the measurement. Also the chain matrix method showed a good match with the magnetic shielding effectiveness measurement of CFRP and consideration of CFRP as a multilayered material with electrically insulated layers was valid. Only disadvantage was the very low shielding effectiveness of CFRP against magnetic fields, this could be enhanced by modifying the material with a high conductive material i.e. copper. Modifications like this, could also be calculated with the chain matrix method.

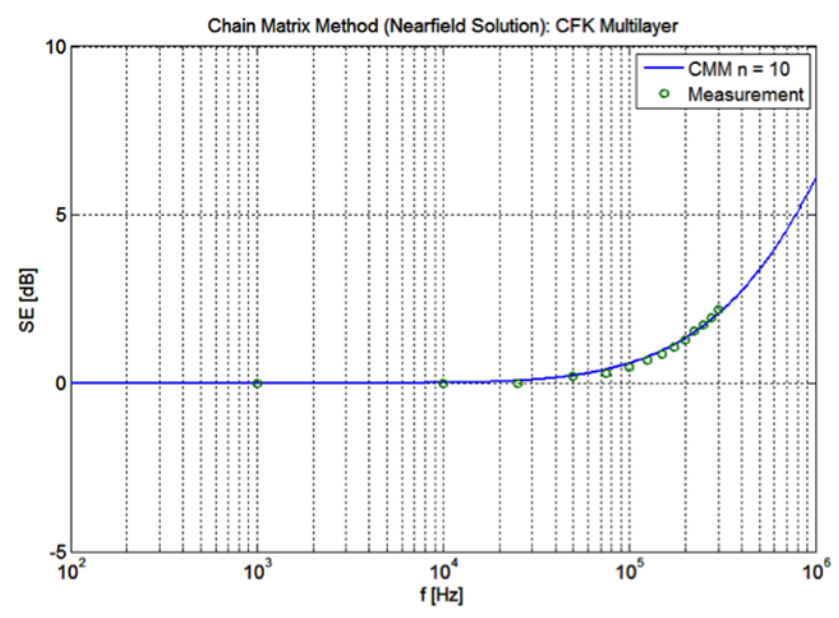

Figure 13. Comparison of chain matrix method with measurement.

Acknowledgements. This contribution was developed within the scope of the project EM4EM (Electromagnetic Reliability of Electronic Systems for Electro Mobility - Subproject: Entwurfs- und EMZ-Messmethodik für EMZ-Analysen auf EV-Gesamtsystemebene) which is funded by the BMBF (Bundesministerium für Bildung und Forschung) under the grant number 16M3092A. The responsibility for this publication is held by the authors only.

Edited by: W. Mathis

Reviewed by: two anonymous referees

\section{References}

Bowler, N.: Theory of four-point direct-current potential drop measurements on a metal plate, Res. Nondestruct. Eval., 17, 29-48, 2006.

Imo, F., Fuchs, C., and Schwab, A. J.: Erweitertes Impedanzkonzept zur Berechnung von geschlossenen Schirmen, Arch. Elektrotech., 76, 437-441, 1993.

Michel, M.: Leistungselektronik: Einführung in Schaltungen und deren Verhalten, Springer-Verlag, 5th edn., 2011.

Moser, J.: Low-Frequency Shielding of a Circular Loop Electromagnetic Field Source, IEEE T. Electromagn. C., 9, 6-18, 1967.

Schelkunoff, S. A.: The Impedance Concept and its Application to Problems of Reflection, Refraction, Shielding and Power Absorption, Bell Syst. Tech. J., 17, 17-48, doi:10.1002/j.15387305.1938.tb00774.x, 1938.

Schwab, A. J. and Kürner, W.: Elektromagnetische Verträglichkeit, VDI-Buch, Springer, 2011.

Vinaricky, E., Keil, A., and Merl, W.: Elektrische Kontakte, Werkstoffe und Anwendungen: Grundlagen, Technologien, Prüfverfahren, Engineering online library, Springer Berlin Heidelberg, 2002.

Wolfsperger, H.: Elektromagnetische Schirmung: Theorie Und Praxisbeispiele, Vdi-buch, Springer, 2008. 